\title{
Fuerza Aérea Colombiana: motor propulsor hacia el espacio ultraterrestre del país hacia un triple desarrollo*
}

| Fecha de recibido: 9 de marzo del 2021 | Fecha de aprobación: 12 de abril del 2021 |

* Este artículo es parte del proyecto de investigación denominado "Proyección del empleo de las capacidades estratégicas aeroespaciales de Colombia para la seguridad y defensa nacional", a cargo del Departamento de Fuerza Aérea de la Escuela Superior de Guerra, inscrito en el grupo de investigación Masa Crítica.

David

Barrero Barrero

Master of Science in Interamerican Defense and Security

Escuela Superior de Guerra

Colombia

Grupo de investigación: Masa Crítica Rol de investigador: teórico y escritura https://orcid.org/0000-0003-0412-1371

$\bowtie$ davidbarrerob@gmail.com

Cómo citar este artículo: Barrero-Barrero, D. (2021). Fuerza Aérea Colombiana: motor propulsor hacia el espacio ultraterrestre del país hacia un tripe desarrollo. Ciencia y Poder Aéreo, 16(1), 87-101, https://doi.org/10.18667/cienciaypoderaereo.707 


\section{Fuerza Aérea Colombiana: motor propulsor hacia el espacio ultraterrestre del país hacia un triple desarrollo}

Resumen: El presente artículo tiene como objetivo reflexionar sobre el enfoque cualitativo a partir del análisis documental, con el fin de demostrar la oportunidad que tiene un país como Colombia, al participar con su Fuerza Aérea en una estructura estatal llamada Comisión Colombiana del Espacio (CCE), y que ha buscado por años alcanzar y consolidar el espacio ultraterrestre para los nacionales. Esa oportunidad se denominó triple desarrollo, debido a los beneficios que tiene actualmente incluir otro escenario como el espacial para aumentar las posibilidades de desarrollo humano. A partir de un análisis documental cualitativo, la pregunta por responder es ¿existen beneficios para Colombia con el uso del espacio ultraterrestre? En ese sentido, los hallazgos del triple desarrollo encontrados fueron: en primer lugar, el factor sostenible, pues organizaciones como Naciones Unidas (UN, por sus siglas en inglés) promueven las posibilidades de reducir las brechas de desigualdad y pobreza con el uso del espacio como fuente de logro; en segundo lugar, la oportunidad económica y social, ya que el espacio genera fuentes de ingreso y eliminación de gastos para Colombia al pagar a otros países el suministro de servicios espaciales; y la tercera oportunidad es el desarrollo tecnológico y la industria. Si Colombia logra consolidar capacidades en estos sentidos, el salto cualitativo hacia la solución de la problemática nacional permitirá hacer un cambio en la historia del país, una nueva hoja de ruta donde el desarrollo y la supervivencia puedan ser moldeables. Lo anterior es parte de la principal conclusión a la cual se espera llegar.

Palabras clave: desarrollo sostenible; economía; economía de la ciencia; espacio; fuerza aérea colombiana; tecnología.

\section{Colombian Air Force: Powering the Country Toward Outer Space within a Triple Development Approach}

Abstract: This work reflects on the participation of Colombia -through the Colombian Air Force- in the Colombian Space Commission (csc) and its importance for the country, considering the efforts made during several years for reaching outer space and consolidating aerospace initiatives. This opportunity has been named "triple development" due to the benefits of including a third scenario, such as space, in the search for new possibilities for human development. Based on a qualitative documentary analysis, the question to be answered is: Does the use of outer space bring any benefits for Colombia? From this, the benefits extracted from the triple development model were: (i) the sustainable factor, since organizations such as the United Nations (UN) promote the possibilities of reducing inequality and poverty gaps through the use of space as a source of development; (ii), economic and social opportunities, considering that space projects generate sources of income and the elimination of expenses for Colombia by paying other countries for the provision of space services; (iii) various technological and industrial development opportunities for the country. If Colombia manages to consolidate capacities in these dimensions, the qualitative leap toward solving certain national problems will allow a change in the country's history, a new roadmap where development and survival can be molded. The above is part of the main conclusion expected to be reached through this study.

Keywords: Sustainable development; economy; economics of science; space; Colombian Air Force; technology.

Resumo: 0 presente artigo tem como objetivo refletir sobre a abordagem qualitativa a partir da análise documental, a fim de demonstrar a oportunidade que tem um país como a Colômbia, ao participar com sua Força Aérea em uma estrutura estatal chamada Comissão Colombiana do Espaço (CCE), e que procurou por anos alcançar e consolidar o espaço ultraterrestre para os nacionais. Essa oportunidade se denominou triplo desenvolvimento, devido aos benefícios que tem atualmente incluir outro cenário como o espacial para aumentar as possibilidades de desenvolvimento humano. A partir de uma análise documental qualitativa, a pergunta a ser respondida é, existem benefícios para a Colômbia com o uso do espaço exterior? Nesse sentido, os achados do triplo desenvolvimento foram: em primeiro lugar, o fator sustentável, pois organizações como as Nações Unidas (NU por sua sigla em inglês) promover as possibilidades de reduzir as brechas das desigualdades e a pobreza através da utilização do espaço como fonte de realização; em segundo lugar, a oportunidade econômica e social, já que o espaço gera fontes de receita e eliminação de despesas para a Colômbia ao pagar a outros países o fornecimento de serviços espaciais; e a terceira oportunidade é o desenvolvimento tecnológico e a indústria. Se a Colômbia conseguir consolidar capacidades neste sentido, o salto qualitativo para a solução da problemática nacional permitirá fazer uma mudança na história do país, um novo guia onde o desenvolvimento e a sobrevivência possam ser moldáveis. Isto faz parte da principal conclusão a que se espera chegar.

Palavras-chave: desenvolvimento sustentável; economia; economia da ciência; espaço; força aérea colombiana; tecnologia. 
Creo y espero sinceramente que, además

de aprovechar los beneficios económicos de democratizar el acceso al espacio para muchos más que los pocos privilegiados que lo hemos explorado hasta ahora, aumentarán en la humanidad, en medida todavía más notable, la buena voluntady la hermandad que resultan de esa experiencia. ${ }^{1}$ Michael López-Alegría²

Colombia ha buscado durante décadas alcanzar el espacio ultraterrestre, conforme a los lineamientos y condiciones de igualdad y soberanía que las Naciones Unidas (UN, por sus siglas en inglés) promueve para todos los Estados miembros del organismo. Actualmente, la Comisión Colombiana del Espacio (CCE) se encuentra en un momento fundamental para generar un cambio y lanzar a Colombia al espacio ultraterrestre, en pro de concretar una hoja de ruta mejor estructurada, bien concebida y respaldada por el Gobierno nacional. La Fuerza Aérea Colombiana (FAC), como miembro de la CCE, contempla en su misión constitucional, además del aire, el espacio como escenario de dominio, en donde ya tiene en órbita un satélite desde el 2018 y avanza en el lanzamiento del segundo.

A pesar de que lo anterior lo materializó de manera autónoma y gracias al valioso recurso humano, su nueva experiencia fortalecerá su doctrina y sus capacidades, las cuales podrán estar a disposición del país, incluyendo seguridad y defensa. Por lo anterior, y mirando más allá, la nueva misión espacial de la FAC será un cohete propulsor que es esencial para que todo el país alcance el espacio, y en el que, en un principio, existe una triple oportunidad de desarrollo para Colombia, como se plantea en este artículo.

1 Traducido de: "It is my belief sincere hope that, in addition to reaping the economic benefits of democratizing access to space to so many more than just the privileged few of us that have gone there thus far, there will be an even more important increase in the goodwill of the brotherhood of man that is a result of the very experience".

2 Astronauta estadounidense de las misiones espaciales Discovery STS-92, Endeavour STS-113 y Soyuz TMA-9/ISS XIV (UNOOSA, 2020).
Acceder al espacio ultraterrestre en el momento contemporáneo que vive la humanidad tiene efectos favorables en todos los sentidos. En el caso específico colombiano, este artículo busca demostrar que el Estado tiene en frente una triple oportunidad de desarrollo que se ha privado de alcanzar, por una multiplicidad de hechos históricos muy poco justificables, y que en esta nueva hoja de ruta la FAC, con los avances que ha realizado en materia espacial, será clave para lograr este objetivo estratégico nacional.

La prioridad para un país en desarrollo como Colombia es reducir las brechas nacionales en términos de tecnología y capacidad industrial, por lo que es necesario hacer alianzas con países estratégicos teniendo en cuenta esas intenciones y procurando el desarrollo, para consolidar y alcanzar una gran variedad de objetivos necesarios para que crezcan la economía y el desarrollo humano, a través del nuevo recurso espacial. Además, sobre la base de los avances tecnológicos a los que se puede acceder, son múltiples los beneficios que trae para el país incorporar el espacio para el logro de los fines esenciales y de los objetivos estratégicos nacionales.

Con el fin de abordar este tema, se proponen tres argumentos en los que se evidencia cómo la FAC debe ser el motor de impulso para la consolidación del espacio ultraterrestre, con el propósito de alcanzar un triple desarrollo. En el primero se aborda la importancia del espacio ultraterrestre para lograr los objetivos de desarrollo sostenible (ODS) planteados por las un, para el beneficio común del planeta. En el segundo se analiza el desarrollo social y económico a partir de las capacidades que tiene trazada la CCE, en la que estos dos aspectos y logros alcanzados hasta el momento por parte de la FAC desempeñan un papel estratégico. En el tercero se examinan las posibilidades de desarrollo tecnológico e industrial que traen como consecuencia cumplir la aspiración colombiana de cara al espacio, en la que se conjugan las nuevas operaciones espaciales de la FAC. Al final, se presentan unas conclusiones alineadas con los argumentos del triple desarrollo que el espacio puede brindarle al país. 


\section{El espacio y el desarrollo sostenible para todos en el planeta}

En el presente apartado se analiza la relación entre el espacio ultraterrestre y el desarrollo sostenible. Ambos se entienden sobre la base de la importancia y la trascendencia que tiene el empleo del escenario espacial con fines pacíficos (Naciones Unidas, 2019, p. 6), en el beneficio común de los seres humanos, como es el caso de los ODs; el fin de estos objetivos es que se logre la igualdad y la equidad para todos los seres humanos, además de buscar el entendimiento de todos para la conservación de la especie humana.

\section{Relación entre espacio ultraterrestre y desarrollo sostenible}

La correspondencia entre estos dos aspectos ha sido un tema de primer orden a lo largo de los años, cuya agenda global la maneja la Comisión sobre la Utilización del Espacio con Fines Pacíficos (UNOOSA por sus siglas en inglés). El eje fundamental para UNOOSA es el de la cooperación internacional en el uso e interés de los Estados por el empleo del espacio respecto a sus propias necesidades, y a partir de la definición anterior, estos fijan sus oportunidades de desarrollo económico, social, científico o tecnológico (Naciones Unidas, 2002, p. 61).

Por otra parte, y en relación con el desarrollo sostenible, entre el 10 y el 17 de junio de 2015, unoosa, en su $58 .^{\circ}$ periodo de sesiones, consideró importante la inclusión del espacio exterior en la nueva agenda en esta materia, además de discutir la sustentabilidad que este asunto debería tener a largo plazo (Naciones Unidas, 2015a). En la mencionada reunión se determinó lo siguiente:

La sostenibilidad a largo plazo debía entenderse como requisito necesario para realizar actividades espaciales, a fin de prevenir toda actividad que pudiera afectar, perjudicar, dañar o destruir los objetos espaciales colocados en órbita o que se dirigieran hacia una órbita; debía impedirse que el espacio se convirtiera en una zona de conflictos entre países o con organizaciones privadas o públicas; debía reconocerse claramente que la colocación de armas en el espacio ultraterrestre, así como todo acto de hostilidad en ese medio, serían incompatibles con la utilización sostenible del espacio ultraterrestre; al adoptarse medidas de mitigación y eliminación de los desechos espaciales se debían tener en cuenta las responsabilidades históricas de los países que realizan actividades espaciales, y en ningún caso debía obligarse a los países con capacidad espacial incipiente a soportar la carga de la eliminación de desechos espaciales ni a contribuir a sufragar los gastos relacionados con las tareas de eliminación (Naciones Unidas, 2015b, p. 30).

De igual forma, en su $62 .^{\circ}$ periodo de sesiones, celebrado entre el 12 y el 21 de junio del 2019, unOoSA hizo "reconocimiento del importante papel de la ciencia y la tecnología espaciales y sus aplicaciones para la implementación de la Agenda 2030 para el DesarroIlo Sostenible, en particular los Objetivos de Desarrollo Sostenible (ODS)" (Naciones Unidas, 2019, p. 37).

Ahora, el nuevo planteamiento de fortalecer los ODS se soporta en la utilización de las tecnologías espaciales, lo que ya es posible mediante el uso de herramientas que contribuyen notoriamente a la detección de áreas propicias para la pesca, la ganadería y la agricultura, al igual que al monitoreo del deshielo glaciar o el de las fuentes de agua dulce en riesgo de contaminación o desaparición, entre muchas otras. Esto permitirá anticipar y tomar las medidas necesarias frente a las adversidades climáticas y los desastres naturales (United Nations Office for Outer Space Affairs).

\section{Los ODS desde el espacio y el plan del gobierno colombiano}

En la tabla 1 se resumen las capacidades espaciales aplicadas a cada uno de los ods según UN, de acuerdo con la Resolución 70/1 "Transformar nuestro mundo: la Agenda 2030 para el Desarrollo Sostenible" (2015c). Lo anterior se fundamenta en las capacidades 
y operaciones espaciales como la Teleobservación de la Tierra (EO, por sus siglas en inglés) y la Geolocalización mediante el uso del Sistema Global de Navegación Satelital (GNSS, por sus siglas en inglés); dos de las estrategias fundamentales que apoyan el cumplimiento de los 17 ods y sus 169 metas. Se espera dar cobertura a todos los países que conforman las UN (United Nations Office for Outer Space Affairs, s.f.-a).

Tabla 1

Uso del espacio ultraterrestre en apoyo a la Agenda 2030

\begin{tabular}{|c|c|c|}
\hline \multicolumn{2}{|r|}{ ODS } & Capacidades espaciales aplicadas a los ODS según UNODC ${ }^{3}$ \\
\hline 1 & Fin de la pobreza & $\begin{array}{l}\text { - Anticipar los desastres naturales y coordinar la provisión de ayuda posterior. } \\
\text { - Óptimo empleo del uso sostenible de los recursos naturales. } \\
\text { - Facilitar el soporte eficiente a las poblaciones vulnerables. } \\
\text { - Mapeo de áreas pobladas y su acceso a servicios básicos. }\end{array}$ \\
\hline 2 & Hambre cero & $\begin{array}{l}\text { - Mejoras en optimización productiva de cultivos a través de un proceso de gestión informado y una mayor eficiencia } \\
\text { con los recursos existentes, incluida la tierra, semillas, fertilizantes, agentes fitosanitarios y agua. } \\
\text { - Mejoras en la gestión del ganado mediante un mejor seguimiento y la identificación de pastos adecuados. }\end{array}$ \\
\hline 3 & $\begin{array}{l}\text { Buena salud y } \\
\text { bienestar }\end{array}$ & $\begin{array}{l}\text { - Estudiar la epidemiología de las enfermedades, al permitir un mayor uso del análisis espacial para identificar } \\
\text { los factores ecológicos, ambientales y de otro tipo que contribuyen a la propagación de enfermedades transmitidas } \\
\text { por vectores. Monitorear los patrones de enfermedades y definir áreas que requieren planificación de control } \\
\text { de enfermedades. } \\
\text { - Abordar problemas relacionados con la asistencia para la visión, la cognición y la discapacidad. } \\
\text { - Monitorear los factores que afectan la salud, así como el bienestar de los seres humanos, como la calidad } \\
\text { del aire y el tráfico. } \\
\text { - Promocionar la salud y, de igual forma, prevenir enfermedades mediante el uso de dispositivos de monitoreo portátiles. } \\
\text { - Habilitación de la atención médica remota. }\end{array}$ \\
\hline 4 & $\begin{array}{l}\text { Educación de } \\
\text { calidad }\end{array}$ & $\begin{array}{l}\text { - Conectividad a internet de alta velocidad y contenido educativo en línea personalizado y entregado por satélite. } \\
\text { - Monitoreo electrónico de asistencia y provisión de incentivos para que los padres reduzcan las tasas de deserción. } \\
\text { - Oportunidades de aprendizaje a distancia, aprendizaje electrónico y aprendizaje permanente para comunidades } \\
\text { remotas y aisladas. }\end{array}$ \\
\hline 5 & Igualdad de género & $\begin{array}{l}\text { - Acceso a una educación de calidad incluso en comunidades remotas y aisladas. } \\
\text { - Apoyo al emprendimiento femenino, a través del acceso a capacitación, infraestructura blanda, información } \\
\text { y seguridad en el ambiente laboral. } \\
\text { - Oportunidades de desarrollo profesional, a menudo dentro de STEM }{ }^{4} \text {. }\end{array}$ \\
\hline 6 & $\begin{array}{l}\text { Agua potable y } \\
\text { saneamiento }\end{array}$ & $\begin{array}{l}\text { - Monitoreo de la calidad del agua. } \\
\text { - Pronóstico meteorológico. } \\
\text { - Acceso a soporte de infraestructura y conocimientos técnicos. }\end{array}$ \\
\hline 7 & $\begin{array}{l}\text { Energía limpia y } \\
\text { asequible }\end{array}$ & $\begin{array}{l}\text { - Monitoreo de infraestructura crítica, particularmente en las redes de energía. } \\
\text { - Sincronización de la red eléctrica. } \\
\text { - Levantamiento sísmico. } \\
\text { - Identificación de sitios óptimos para la producción de energía renovable. } \\
\text { - Previsión para producir energía solar y eólica para estimar la cantidad de energía necesaria de otras fuentes. }\end{array}$ \\
\hline 8 & $\begin{array}{l}\text { Trabajo decente } \\
\text { y crecimiento } \\
\text { económico }\end{array}$ & $\begin{array}{l}\text { - Las economías mundiales y el crecimiento del PIB, que contribuyen a una variedad de sectores, en particular la } \\
\text { prestación de servicios públicos, la banca y las finanzas, la agricultura y las comunicaciones. } \\
\text { - Monitoreo de trabajadores solitarios, estableciendo entornos de trabajo seguros y protegidos. }\end{array}$ \\
\hline 9 & $\begin{array}{l}\text { Industria, } \\
\text { innovación e } \\
\text { infraestructura }\end{array}$ & $\begin{array}{l}\text { - Mapeo y monitoreo de la infraestructura, incluido el mantenimiento de la infraestructura vial en entornos rurales, } \\
\text { donde la tecnología más confiable es la basada en satélites. } \\
\text { - Topografía de la construcción mediante la automatización de máquinas. } \\
\text { - Movilidad inteligente, por ejemplo, menor consumo de combustible mediante una planificación y un seguimiento } \\
\text { más inteligente del comportamiento de conducción. }\end{array}$ \\
\hline
\end{tabular}

3 Oficina de Naciones Unidas contra la Droga y el Delito.

4 Sigla para citar las disciplinas: "Science, Technology, Engineering and

Mathematics” (ciencia, tecnología, ingeniería y matemáticas). 


\begin{tabular}{|c|c|c|}
\hline 10 & $\begin{array}{l}\text { Reducir las } \\
\text { desigualdades }\end{array}$ & $\begin{array}{l}\text { - Conectividad en áreas remotas y aisladas. } \\
\text { - Participación remota en procesos democráticos. } \\
\text { - Acceso confiable a la información. }\end{array}$ \\
\hline 12 & $\begin{array}{l}\text { Producción } \\
\text { y consumo } \\
\text { responsables }\end{array}$ & $\begin{array}{l}\text { - Manejo de recursos naturales. } \\
\text { - Trazabilidad de alimentos y mercancías peligrosas. } \\
\text { - Monitoreo del tráfico de especies en peligro y productos de la esclavitud humana. } \\
\text { - Agricultura inteligente mediante la combinación de observación terrestre, telecomunicaciones por satélite y sistemas } \\
\text { de navegación global satelital. } \\
\text { - Los productos derivados de la utilización de recursos in situ (ISRU), como las tecnologías de impresión 3D para crear } \\
\text { estructuras en órbita podrían tener aplicaciones en la Tierra. }\end{array}$ \\
\hline 13 & Acción climática & $\begin{array}{l}\text { - Monitoreo del cambio climático. } \\
\text { - Predicción del tiempo. } \\
\text { - Gestión de desastres. } \\
\text { - Operaciones de búsqueda y salvamento. }\end{array}$ \\
\hline 14 & Vida submarina & $\begin{array}{l}\text { - Mapeo y monitoreo de áreas naturales y protegidas. } \\
\text { - Seguimiento y navegación de buques pesqueros. } \\
\text { - Seguimiento de la pesca ilegal no reglamentada y no declarada. } \\
\text { - Trazabilidad de productos pesqueros - especies en peligro de extinción, explotación de recursos pesqueros-. } \\
\text { - Evaluación y seguimiento de los recursos marinos y costeros. } \\
\text { - Monitoreo del cambio climático, particularmente la temperatura del agua. } \\
\text { - Identificación de floraciones de algas. }\end{array}$ \\
\hline 15 & Vida en la Tierra & $\begin{array}{l}\text { - Monitoreo biogeofísico de la superficie terrestre. } \\
\text { - Monitoreo de la biodiversidad terrestre. } \\
\text { - Seguimiento de la caza furtiva e identificación de rutas de contrabando. }\end{array}$ \\
\hline 16 & $\begin{array}{l}\text { Paz, justicia e } \\
\text { instituciones } \\
\text { sólidas }\end{array}$ & $\begin{array}{l}\text { - Monitoreo de conflictos. } \\
\text { - Permitir la participación de comunidades remotas y aisladas en procesos democráticos. } \\
\text { - Cumplimiento de la legislación. } \\
\text { - Acceso a información confiable. }\end{array}$ \\
\hline 17 & $\begin{array}{l}\text { Alianzas para los } \\
\text { Objetivos }\end{array}$ & $\begin{array}{l}\text { - Iniciativas de cooperación internacional. } \\
\text { - Intercambio de datos e información. } \\
\text { - Bases de datos de código abierto. } \\
\text { - Intercambio de infraestructura e intercambio de conocimientos técnicos. }\end{array}$ \\
\hline
\end{tabular}

Fuente: elaboración propia a partir de la Resolución de las Naciones Unidas 70/1 “Transformar nuestro mundo: la Agenda 2030 para el Desarrollo Sostenible” (2015).

Esta nueva hoja de ruta que integra espacio y desarrollo se centra en la solidaridad y compromiso de las naciones para dar cumplimiento no solo a la agenda, sino a la inclusión de las capacidades espaciales de los países que han desarrollado estas últimas, al servicio y beneficio de los países que trabajan por alcanzar algún desarrollo espacial, y en general, para el beneficio de la humanidad. Por lo tanto, uno de los aspectos importantes para el logro de los ODS son las tecnologías espaciales inclusivas, las cuales deberán, entre otras, "contribuir a prevenir que las personas caigan por debajo del umbral de la pobreza y ayudar a orientar el apoyo específico a quienes lo necesitan" (United Nations Office for Outer Space Affairs, s. f. -b).

Tras el análisis del horizonte trazado por UN, al incluir el uso de las capacidades espaciales en el cumplimiento de la Agenda 2030, y teniendo en cuenta que resulta viable e incluyente para todos los países del mundo, en Colombia se decidió integrar a los oDs en el actual plan de Gobierno. En el denominado "Plan Nacional de Desarrollo 2018-2022. Pacto por Colombia, pacto por la equidad" (Congreso de Colombia, 2018), 
se encuentran las estrategias y programas que impulsan la transformación digital y tecnológica, así como la implementación de "una política nacional para desarrollar el sector espacial” (Congreso de Colombia, 2018, p. 727), la cual incluye como oportunidad el acceso y uso de las tecnologías espaciales y satelitales. Además, se planteó el combate contra las amenazas trasnacionales, en cooperación con otros países, en diferentes escenarios que incluyen el espacial (Congreso de Colombia, 2018, p. 73).

Lo anterior implica llevar a Colombia a ubicarse en todos los escenarios: terrestre, marítimo, aéreo, ciberespacial y espacial; en el caso de este último, se busca incluir al país en esas oportunidades que le permitan generar un triple desarrollo. La CCE trabaja en este tema con el fin de delinear un mejor y próspero escenario para Colombia. Es así como, dentro de su estructura organizacional, la FAC se ha constituido como uno de los motores para el logro de las aspiraciones por alcanzar el objetivo espacial, acudiendo a sus adelantos en esta materia, dándole una base firme a la CCE para que cumplan con sus objetivos.

\section{La necesidad de una economía espacial para Colombia}

La globalización abrió las puertas de una nueva economía mundial, con lo que logró la integración de las economías nacionales, así como la apertura de mercados, la interdependencia económica, la reducción de barreras comerciales y la rapidez de las negociaciones, hechos que permiten dar solución a muchas necesidades locales en materia económica. En este sentido, la inclusión de una economía espacial es contundente y necesaria en el logro de objetivos nacionales con miras al cumplimiento de los ODS.

De hecho, el crecimiento económico moderno durante los últimos doscientos cincuenta años se ha modelado y modificado debido a la multiplicidad e importancia de factores que intervienen en la economía global, a causa de la evolución y desarrollo de la tecnología (Sachs, 2015, p. 158-159). Por ello, al hablar de un triple desarrollo, no se aísla a cada uno de los desarrollos planteados. En este caso, la tecnología requiere de la economía para el desarrollo, pero la economía necesita a la tecnología para obtener mayores ganancias en menor tiempo y esfuerzo.

Respecto al uso del espacio con fines económicos, en el libro Mirando hacia las estrellas: una constante necesidad humana, se afirma lo siguiente:

Las actividades espaciales comerciales más rentables son aquellas que proporcionan comunicaciones rápidas en todo el mundo. Ya sea que se trate de servicios de navegación y temporización de los satélites GPS, transmisiones directas de TV, enlaces de terminales de muy pequeña apertura de las compañías de tarjetas de crédito, o comercio financiero electrónico, el sistema económico global ahora está vinculado a través de satélites y capacidades espaciales. (Álvarez, Jaimes \& Paredes, 2019, p. 335)

Para entender mejor este tema en cifras, según el "Yearbook on Space Policy 2017" (Burger, 2017), en el 2017, ya había 50 países con al menos una organización dedicada a las actividades espaciales, de los cuales nueve estados tenían un presupuesto superior a un billón de dólares (p. 54); de hecho, la actividad espacial global en el mismo año fue de 383,51 mil millones USD siendo el 80,1\% correspondiente a los ingresos totales del sector espacial comercial (307, 32 mil millones USD), incluidos los sectores público y privado, lo cual revela las oportunidades económicas que deja la actividad espacial en términos de economía (Burger, 2017, p. 54). Estos datos evidencian el hecho de que "el Gobierno tiene un papel absolutamente crucial en el desarrollo económico de un país" (Sachs, 2015, p. 196) y, en consecuencia, debe buscar las alternativas que permitan lograrlo. El espacio ultraterrestre es quizá la mejor opción a futuro.

Adicionalmente, en 2018 la infraestructura comercial y la industrial generaron 95,87 mil millones USD gracias a actividades como la fabricación de satélites comerciales, la provisión de servicios de lanzamiento y la provisión de seguros de activos espaciales para el lanzamiento de satélites, además de las empresas de explotación de satélites (Burger, 2017, p. 54). En ese 
sentido, hay un crecimiento sincronizado en todas las principales economías desarrolladas (p. 3).

Ahora bien, una vez analizado el plan del Gobierno colombiano para integrar el uso del espacio ultraterrestre y la Agenda Global para el 2030, también es importante hacer una revisión de la estrategia nacional. El punto de partida son los documentos elaborados por el Consejo Nacional de Política Económica y Social (CONPES), pues a partir de estos se establece la hoja de ruta hacia un próspero desarrollo en Colombia, tanto en lo económico como en lo social, a través de la explotación de las capacidades y beneficios que puede brindar el espacio.

De acuerdo con la Organización para la Cooperación y el Desarrollo Económicos (OECD, por sus siglas en inglés) (citado en Álvarez et al., 2019), la economía espacial es:

La gama completa de actividades y el uso de recursos que crean y proporcionan valor y beneficios a los seres humanos en el curso de la exploración, comprensión, gestión y utilización del espacio. Por tanto, incluye a todos los actores públicos y privados involucrados en el desarrollo, la provisión y el uso de productos y servicios relacionados con el espacio, que van desde la investigación y el desarrollo, la fabricación y el uso de infraestructura espacial (estaciones terrestres, vehículos de lanzamiento y satélites), hasta aplicaciones habilitadas para el espacio (equipos de navegación, teléfonos satelitales, servicios meteorológicos, etc.), y la ciencia conocimiento generada por tales actividades. De ello se deduce que la economía espacial va mucho más allá del sector espacial en sí mismo, ya que también comprende los impactos cada vez más penetrantes y continuamente cambiantes tanto cuantitativos como cualitativos de los productos, servicios y conocimientos derivados del espacio sobre la economía y la sociedad (p. 339).

En este sentido, los adelantos ya desarrollados por la FAC, específicamente la puesta en órbita del FACSAT-1 - su primer satélite espacial instalado el 28 de noviembre del 2018-, no constituyen simples hechos aislados y desapercibidos para la sociedad colombiana (Fuerza Área Colombiana, 2019). El hecho de que el país haya logrado colocar un satélite en órbita, por medio de uno de sus organismos que conforma la CCE, es un paso firme que requiere la participación de la Nación alrededor del éxito y de sus beneficios.

Si se analiza lo económico, la información que en el futuro suministre la FAC a la CCE, la academia y demás órganos que la requieran, será el primer inicio de reducción en gastos por efecto de pago de servicios espaciales $y$, a su vez, el inicio de actividades espaciales con beneficio económico para Colombia.

Así mismo, el FACSAT-1 se unió al primer pico satélite colombiano en el espacio ultraterrestre, denominado LIBERTAD-1 de la Universidad Sergio Arboleda, puesto en órbita el 17 de abril del 2007 (Joya, s.f.). Estos dos registros demuestran la capacidad de las instituciones y la iniciativa de su gente, lo cual debe ser apoyado para lograr los resultados ya mencionados. Según la "Estrategia para el desarrollo aéreo y espacial de la Fuerza Aérea Colombiana 2042" (2020), la FAC tiene previsto para el 2022 la adquisición de su segundo satélite FACSAT-2, este hecho fue analizado, en términos de lo tecnológico y lo económico, por el doctor Raúl Andrés Joya Olarte, director del Observatorio Astronómico de la Universidad Sergio Arboleda, quien afirmó:

Esta segunda experiencia tiene que ir ya más apalancada y deberá fundamentarse en los servicios que prestaría un satélite de observación de la Tierra. En este orden de ideas, recoger las necesidades que no solamente tenga la Fuerza, porque seguramente las prestaciones que se requerirían para servicios de inteligencia, seguridad y defensa son bastante altas y los recursos desconozco si estarán a esa altura.

[...] el nuevo satélite debe tener las prestaciones [...] a nivel académico y a nivel de algunas instituciones del Estado, que serían como: el Servicio Meteorológico (IDEAM), el Instituto Geográfico Agustín Codazzi que complementarían tareas muy importantes como la del catastro multipropósito, la agricultura de precisión, la minería ilegal o la prevención de desastres naturales (R. Joya, entrevista virtual, 30 de junio del 2020). 
Es importante aclarar que la estrategia FACSAT es mucho más que contar la historia de un lanzamiento y el trabajo que se adelanta para un segundo. Por el contrario, el objetivo de la FAC es consolidar seis de estos nanosatélites en órbita. Todos ellos a "disposición de la nación" (Urbina Carrero, 2020. p. 207), lo que debe motivar más aún a un país lleno de necesidades, buscar en su interior, el motor de lanzamiento al triple desarrollo espacial que formula el presente documento.

En suma, es importante y necesario fortalecer el proceso y el modelo adoptado por el cual Colombia, a través de la Universidad Sergio Arboleda y la FAC, ha alcanzado el espacio. De hecho, en el actual CONPES 3.983 se evidencian obligaciones para cada miembro de la CCE, por ejemplo, las "Líneas de Acción" trazadas para la FAC, que incluyen un trabajo orientado hacia el desarrollo económico, social y tecnológico nacional; en esa medida, van más allá de los compromisos propios de seguridad y defensa, sin desconocer su misión de contribuir a los fines del Estado (Fuerza Aérea Colombiana, s.f.).

\section{Estrategia nacional en materia espacial}

De acuerdo con lo que ha establecido el Gobierno nacional, el objetivo de la política espacial colombiana es "generar las condiciones habilitantes y el entorno institucional adecuado para que, con una visión de largo plazo", se active el ecosistema espacial contribuyendo a la productividad y competitividad de Colombia (C. Corredor, comunicación personal, 30 de junio del 2020; Comisión Colombiana del Espacio, 2020). En este sentido, en el CONPES se crearon las "líneas estratégicas para el desarrollo del sector [...] estableciendo una agenda de cooperación internacional, así como la adaptación del marco normativo del país” (2020, p. 4).

Después de varias décadas sin mayores avances al respecto, el 13 de enero de 2020 el Gobierno nacional aprobó el CONPES 3.983, denominado "Política de Desarrollo Espacial: condiciones habilitantes para el impulso de la competitividad nacional" (2020). En dicho documento fueron consideradas "las condiciones [...] para que las tecnologías espaciales sean un impulsor de la productividad, la diversificación y la sofisticación de la economía colombiana" (p. 3). Para lograrlo, la cCE consideró que la "iniciativa pública tiene como fin último promover el crecimiento económico y el desarrollo social del país" (p. 3). Precisamente el objetivo de la política es:

Plantear una política pública desde la cual se generen las condiciones habilitantes para que el país pueda explotar el sector espacial para mejorar la productividad, la diversificación y la sofisticación del aparato productivo colombiano, en línea con el Documento CONPES 3.866 Política Nacional de Desarrollo Productivo (CONPES 3.983, 2020, p. 3).

¿Qué esperar entonces de esta política de gobierno? La Vicepresidencia de la República, a cargo de Marta Lucía Ramírez, es la responsable de los asuntos espaciales para Colombia, así como de la reactivación de la CCE. Durante su intervención en la Semana Mundial del Espacio 2020 (wSw), la vicepresidenta afirmó que el Gobierno nacional y la CCE se dieron:

\begin{abstract}
A la tarea de diseñar una política pública para el sector espacial, que contribuya al crecimiento sostenible del país en dos vías: impulsando el desarrollo de industrias nacionales para la generación de una economía intensiva en conocimiento, la creación de empleo calificado, y la diversificación del aparato productivo del país; y promoviendo el cierre de brechas socioeconómicas, con una mayor cobertura de servicios públicos estratégicos (Vicepresidencia Colombia, 2020).
\end{abstract}

\section{El modelo espacial norteamericano}

Una vez visto el horizonte que está trazando Colombia con miras al espacio, y para adecuar una estrategia espacial que contribuya a este propósito, la FAC ha revisado algunos modelos regionales y experiencias de países amigos para ajustar su propio plan; el ejemplo norteamericano es un interesante referente. La misión de la recientemente creada Fuerza Aérea Espacial de los Estados Unidos (AFSPC, por sus siglas en inglés) es la de "proporcionar capacidades espaciales 
resistentes, defendibles y asequibles para la Fuerza Aérea, la Fuerza Conjunta y la Nación"5.

La transición del aire al espacio ha sido un tema sencillo y creativo para la Fuerza Aérea de los Estados Unidos (USAF, por sus siglas en inglés). Corresponde a la decimocuarta fuerza aérea americana la tarea de "proporcionar capacidades espaciales para la lucha conjunta a través de las misiones operativas de elevación espacial; posición, navegación y cronometraje; comunicaciones por satélite; alerta de misiles y control espacial"' (Air Space Force Command, 2020).

En el documento "America's Air Force: A Call to the Future" (United States Air Force, 2014), la USAF determinó que para los próximos años, "el espacio continuará como un dominio ${ }^{7}$ vital para la economía global, ya que proporciona comunicaciones críticas; datos de posición, navegación y sincronización; e imágenes que han abierto la puerta a avances notables" (United States Air Force, 2014, p. 7), precisamente donde las nuevas tecnologías deberán lograr las oportunidades que alterarán favorablemente la economía (United States Air Force, 2014, p. 7).

A pesar de lo anterior, el dominio del aire sigue siendo indispensable para las actividades de defensa y seguridad, así como para el aporte a la economía mundial. Además, está claro que el ciberespacio ha venido creciendo y lo seguirá haciendo como un dominio crítico en el cual fluye un considerado volumen de información en velocidades cada vez mayores. Respecto al dominio espacial, con mayor razón, este será vital para la economía global (United States Air Force, 2014, p. 7), gracias a su rápida capacidad de alterar la economía de las naciones.

5 Traducido de: "Provide resilient, defendable and affordable space capabilities for the Air Force, Joint Force and the Nation".

6 Traducido de: "provides space capabilities for the joint fight through the operational missions of spacelift; position, navigation and timing; satellite communications; missile warning and space control".

7 En el sentido simple, dominio hace referencia, entre otros, al acceso a la información y a un bien público mundial (Tesauro de la Unesco, 2020).

8 Traducido de: "Space will continue as a vital domain for the global economy, as it provides critical communications; position, navigation, and timing data; and imagery that have opened the door to remarkable advancements".
Cabe agregar que para la participación en las operaciones espaciales norteamericanas la ASPFC ha generado un desarrollo integral y estructural al interior de la organización, además de la generación de nuevas capacidades. Un trabajo que se ha venido ejecutando desde el $1 .^{\circ}$ de septiembre de 1982 (Air Space Force Command, s.f.).

Ahora bien, algunas de las capacidades que contribuyen a las misiones y operaciones espaciales para el triple desarrollo son: el empleo de radares terrestres; el monitoreo del lanzamiento de misiles balísticos en todo el mundo, con el fin de proteger al territorio estadounidense de agresiones (Air Spcae Force Command, s.f.); las instalaciones para las bases de lanzamiento con los servicios necesarios; el control para la seguridad de las misiones en coordinación con el Departamento de Defensa y la Nasa; y la cobertura global para las misiones y enlaces de comunicaciones. Lo anterior permite integrar una infraestructura a la seguridad, monitoreo, soporte necesario para comando y control, seguridad en los lanzamientos e infraestructura para las misiones espaciales (Air Space Force Command, s. f.).

La revisión del modelo de la USAF en su camino hacia el espacio permite comparar y afirmar que Colombia, a través de su CCE y de la FAC, tiene el reto y la oportunidad de transformar la Fuerza Aérea desde un servicio centrado en la plataforma aérea, donde el espacio y el cibernético no tienen protagonismo, a una organización verdaderamente centrada en el conocimiento y con multidominio ${ }^{9}$ (Zacharias, 2019, p. XVIII). Este concepto de multidominio hace referencia a los escenarios terrestres, marítimos, aéreos, espaciales - analizados en el presente documento-y ciberespaciales. Este último es más complejo, debido a su composición de sistemas electrónicos y en red que utilizan energía electromagnética, y que pueden relacionarse

9 Adaptado del texto original: "We have a unique opportunity to transform the Air Force from an air platform - centric service, where space and cyber often take a back seat, to a truly multidomain and knowledge-centric organization. By delivering autonomous systems to the warfighter by way of a Knowledge". 
directamente con cada uno de los anteriores y explotar mayores capacidades (Parkinson, 2019).

\section{Desarrollo tecnológico e industrial espacial}

Las primeras imágenes del planeta fueron tomadas a 100 kilómetros de altitud en 1947, desde un cohete alemán V-2 confiscado por los americanos desde la Segunda Guerra Mundial y acondicionado para esta labor. Las mencionadas fotografías corresponden a la zona de Nuevo México, California, y, aunque fueron reveladas hasta 1984 (Rodríguez, 2020), son un hecho histórico, no solo por el desarrollo tecnológico, sino porque satisficieron la inmensa necesidad que, desde la época de Nicolás Copérnico, ha tenido la humanidad de estudiar en dónde vivimos y qué nos hace, hasta ahora, únicos; un planeta que nos proporciona todo para vivir, pero igualmente complejo, por lo que es necesario evaluarlo cada día y sacar el mejor beneficio para toda la naturaleza.

Quienes han logrado acceder al espacio ultraterrestre han alcanzado conocimiento y desarrollo en ciencia y tecnología, y posteriormente, han explotado estas capacidades y generado una economía productiva, a partir de la venta de servicios espaciales a otros países. Invertir en este proceso implica generar mayores conocimientos, lo que promueve el desarrollo y la integración de la tecnología y la industria espacial a sus activos sociales y económicos, incluyendo la educación del futuro.

En este contexto, la Innovación, el Desarrollo y la Iniciativa ( $(+D+1)$ son una combinación determinante para una Colombia que necesita oportunidades de triple desarrollo, con la inclusión del espacio a sus activos. Las oportunidades de tecnología e industria previstas en el CONPES 3.983 le permitirían a Colombia aspirar a una mejor calidad de vida para todos en el país, con el aumento de academia, trabajo, salud y productividad, no solo local, sino con proyección global.

De acuerdo con lo anterior, se puede afirmar que sin la tecnología es casi imposible lograr el desarrollo sostenible y los desarrollos económicos y sociales mencionados, ya que esta constituye el elemento gestor de la realidad espacial de largo plazo para el país; un camino que han seguido algunas naciones del mundo y con el que han conseguido resultados de primer orden.

\section{Tecnología en el espacio para el uso y beneficio común}

El 13 de diciembre de 1958, las Un determinaron que el espacio sería de uso común para todos y que tendría fines pacíficos bajo el principio de "igualdad soberana [...] [estimulando] enérgicamente la más completa exploración y explotación del espacio ultraterrestre en beneficio de la humanidad" (p. 5). Desde entonces, le solicitó al primer grupo de 18 países presentar un informe que, entre otras actividades, debía mostrar "la esfera en que se podría emprender adecuadamente, bajo los auspicios de las Naciones Unidas, una cooperación y programas internacionales [...] cual fuere su grado de desarrollo económico o científico" (p. 6). Por ese motivo, unOoSA ha buscado trabajar con el fin de ayudar a todos, pero "especialmente [a] los países en desarrollo, a acceder y valerse como beneficiarios de lo que el espacio puede proporcionar para alcanzar el desarrollo sostenible (United Nations Office for Outer Space Affairs, s. f. -a).

Es así como en materia de cooperación y desarrollo, la tecnología que se envía al espacio genera aportes fundamentales - algunos de valor estratégico-, puesto que esta tecnología contribuye a comprender problemas complejos como el calentamiento global $y$, a partir de allí, se les facilita a las sociedades la posibilidad de mitigar y anticipar consecuencias (Naciones Unidas, 2015a); quizás es esa la principal misión de nuestro futuro inmediato, a fin de determinar los cambios y los daños que el mismo ser humano le está ocasionando a la Tierra. Asimismo, se puede obtener información para prevenir riesgos, desastres naturales y de origen humano, rastreo al deshielo, preservación de especies animales en riesgo e incluso anticipación de enfermedades.

Estas tecnologías, de aplicación civil y militar o de uso dual, representan para los países exploradores del espacio una oferta amplia de conocimientos: recursos naturales, territorio, clima, soberanía, 
medicina, prevención de desastres, desarrollo sostenible, desarrollo humano, seguimiento de actividades del terrorismo y crimen trasnacional. A su vez, el desarrollo espacial hoy en día es una actividad en la que la inversión público-privada produce un beneficio mayormente amplio y próspero para los países. Por lo tanto, permite convertir al espacio en una empresa global en la que la participación mixta de estos capitales ayuda de manera rápida a un crecimiento favorable y seguro (Álvarez et al., p. 331). Esta es la denominada economía espacial de la OCDE, definida anteriormente, cuyo fin se ha planteado a largo plazo e implica la formalización de una alianza entre el Estado y empresa privada (OCDE, 2014b; OCDE, 2016; Congreso de Colombia, 2018, p. 727).

De acuerdo con lo expresado por Clayton Chung en 2001 en su libro Aerospace Power in the Twenty-First Century. A Basic Primer, en donde trató de hacer énfasis en las ya relacionadas bondades de las tecnologías espaciales, el empleo de las siguientes capacidades:

Los programas de alerta temprana, meteorológicos y otros programas de apoyo [ha] demostrado el valor y la necesidad de incorporar capacidades basadas en el espacio para operaciones aéreas, así como para acciones terrestres y marítimas. Los avances tecnológicos en propulsión, materiales, electrónica, computadoras, comunicaciones y otros descubrimientos han ayudado a despertar el interés en los sistemas espaciales. La dependencia de la constelación de satélites del Sistema de Posicionamiento Global (GPS) para la navegación y la entrega de armas por parte de todos los servicios ha aumentado la necesidad de operar y mejorar continuamente el sistema. Sin embargo, una limitación del poder espacial es el proceso costoso y sensible al tiempo de enviar un sistema espacial a la órbita ${ }^{10}$ (Chung, 2001, p. 298).

10 Traducido de: "Early warning, weather, and other support programs have illustrated the value and necessity of incorporating space-based capabilities for aerial operations as well as ground and maritime actions. Technological advances in propulsion, materials, electronics, computers, communications, and other discoveries have helped ignite interest in space systems. Reliance upon the Global Positioning System (GPS) satellite constellation for navigation and weapons delivery by all services has increased the need to continually operate and improve the system. One
Por lo tanto, acceder a este nuevo poder espacial para un país como Colombia permitirá la evolución de su desarrollo tecnológico flexible, base de un triple desarrollo, a pesar de que persisten algunas preguntas sobre el uso de armas en el espacio y todo lo que rodea una eventual capacidad bélica por parte de países como Estados Unidos, Francia y China. Puede que ese momento esté cerca o por lo menos que sea probable, sin embargo, UNOOSA trabaja en reducir estos riesgos y en mantener al espacio libre de ser empleado contra el ser humano. Afortunadamente, el uso del espacio, por ahora, se ajusta a los propósitos de uso terrestre y beneficio común (Chung, 2001, p. 299).

A propósito de lo anterior, y retomando el Informe de la Comisión sobre unoosA, expedido en su $62 .^{\circ}$ periodo de sesiones, queda claro el uso común del espacio para la humanidad, del cual se excluye su empleo para temas diferentes al beneficio que representa. Al respecto, la Comisión:

Tomó nota de la contribución que hacían la tecnología espacial y sus aplicaciones, así como la información y los datos obtenidos desde el espacio, al desarrollo sostenible, en particular en cuanto a mejorar la formulación y la posterior aplicación de las políticas y los programas de acción relacionados con la protección del medio ambiente, la gestión de tierras y recursos hídricos, el desarrollo urbano y rural, los ecosistemas marinos y costeros, la atención de la salud, el cambio climático, la reducción de los riesgos de desastre y la respuesta de emergencia, la energía,la infraestructura, la navegación, la vigilancia sísmica, la gestión de los recursos naturales, las nieves y los glaciares, la biodiversidad, la agricultura y la seguridad alimentaria (Naciones Unidas, 2019, p. 37).

\section{Tecnoestrategia colombiana}

Las condiciones habilitantes mencionadas en el CONPES 3.983 (2020, p. 3) para el desarrollo productivo de la Nación parten de una base tecnológica espacial

limitation of space power, however, is the time-sensitive and costly process of sending a space system into orbit". 
como motor gestor del triple desarrollo tratado en este documento. Al referirse al tema de los lineamientos referentes para la observación de la Tierra a nivel nacional, los cuales deben ser viables e incluir la realización del diseño de un Programa Satelital para Colombia (2020), el Gobierno se alinea con lo planteado por UNOOSA anteriormente, y propone utilizar en Colombia:

Las tecnologías espaciales para la educación, la salud, la vigilancia ambiental, la exploración y gestión de recursos naturales, la atención de desastres, el monitoreo de los usos del suelo, las previsiones meteorológicas, el cambio climático, el transporte terrestre, la navegación marítima, fluvial y aérea y las telecomunicaciones, entre otras temáticas, orientadas al desarrollo económico, cultural y social sostenible (2020, p. 5).

En este sentido, el triple desarrollo incorpora también a la educación como materia prima para el fomento de temas espaciales, gracias a los avances tecnológicos. Para determinar y alcanzar el tamaño de fuerza de la CCE y especialmente el de la FAC, Raúl Joya (comunicación personal, 30 de junio del 2020) hizo referencia a la necesidad de incorporar personal administrativo y abogados especializados en el derecho operacional. Esto se sumaría a la integración de personas con diferentes especialidades y carreras profesionales afines a la ingeniería espacial, como la mecatrónica, la electrónica, las telecomunicaciones, el área eléctrica y la física, por citar tan solo unas pocas. Todas ellas se mantienen alrededor de la construcción de la tecnología base para lanzar a Colombia al espacio, no sin dejar de citar a aquellas que se relacionan con la difusión a través de los medios de comunicación, así como el convencimiento de las actividades que desarrolla la CCE al pueblo colombiano.

El concepto estratégico que tiene la FAC respecto al "acceso y uso de las tecnologías satelitales como una capacidad inherente y distintiva de la institución" (Quiroga, 2019, p. 24) brinda la garantía de forjar un futuro espacial, soportado en la tecnología y la industrialización espacial al interior colombiano.
Finalmente, las capacidades tecnológicas abren camino en todas las direcciones para el desarrollo. Colombia ha iniciado un camino muy interesante $y$, sobre todo, ha decidido cumplir la estrategia que permite adquirir la tecnología espacial necesaria para gestionar la transformación política, social y económica que fomente este nuevo camino de éxito hacia el espacio, lo que permite asegurar un mejor futuro para todos los colombianos.

\section{Conclusiones}

Desde el principio, este documento demostró de qué manera un país como Colombia puede alcanzar el espacio ultraterrestre y servirse de sus capacidades de desarrollo, con la participación activa de la FAC como miembro de la CCE, y como motor de propulsión que impulsa el logro de este objetivo tan necesario e importante para mejorar el desarrollo del país en tres aspectos llamados triple desarrollo: el sostenible, el económico y social y, finalmente, el tecnológico e industrial. Con el fin de argumentar esta idea, se desarrolló individualmente un apartado sobre cada uno de ellos para visibilizar oportunidades y capacidades en las que la FAC se constituye como el motor de propulsión del país.

Las UN ya han establecido un escenario global de inclusión, en el que el espacio ultraterrestre se pone al servicio de la humanidad y contribuye al cumplimiento de los ODS. Aun en el siglo XXI hay países rezagados que no tienen la oportunidad de este beneficio. Sin embargo, UNOOSA ha hecho posible este acceso al proponer que los países que han logrado llegar al espacio ayuden a los demás Estados a lograr este mismo objetivo; por consiguiente, la hoja de ruta trazada permitirá que poco a poco, la humanidad amplíe las expectativas de alcanzar la Agenda 2030.

En este sentido, y con la participación de un capital mixto que posibilite el alcance de una economía espacial, las posibilidades de un segundo desarrollo, de tipo económico y social, serán tangibles gracias al aporte de las capacidades de cada institución de la CCE, y dentro de ella, la FAC, una entidad que ha avanzado en estudios espaciales y en la puesta en órbita de 
un satélite. En el futuro será la teleobservación una de las primeras capacidades por alcanzar, pues esta beneficiará desde el primer momento a todos los sectores públicos y privados, cuya inversión en conjunto genera la sinergia del éxito.

Cabe aclarar que el logro de las capacidades tecnológicas necesarias para llevar a Colombia al espacio ultraterrestre será el tercer desarrollo estratégico para la Nación. Las tecnologías espaciales abrirán las puertas del triple desarrollo colombiano a partir de su acceso a este escenario tan imprescindible para forjar el futuro que Colombia requiere y merece. Con su misión, la FAC aporta capacidades que van más allá de la defensa y la seguridad. Los fines esenciales del Estado se encuentran inscritos en su misión, y además determinan que el aire, el espacio y el ciberespacio son el teatro de operaciones en el que la FAc traza su dominio para Colombia.

En suma, el presente documento resalta la riqueza teórica y las capacidades de la FAC en su condición de miembro de la CCE, como cohete propulsor de Colombia hacia el espacio. Más allá de los beneficios en seguridad y defensa, su aporte es el compromiso con los fines esenciales del Estado en pro de un triple desarrollo.

El aporte teórico de este escrito resalta las cualidades y capacidades de la FAC, la cual ha hecho una gran transformación con miras hacia el futuro, donde ha ampliado su espectro de acción al espacio y el ciberespacio. Ante este suceso, es oportuno valerse de este nuevo horizonte y de la experiencia de tener en órbita un satélite propio, lo que se constituye en la base y columna vertebral para que, a través de la CCE, se consolide esa necesidad de triple desarrollo para el país.

Se recomienda que el Gobierno respalde el lanzamiento del próximo satélite de la FAC, en el que deben participar todos los miembros de la Comisión, y cuyo aporte será compatible con las aspiraciones de triple desarrollo para Colombia. Finalmente, en un escenario indefinible como el espacio no debe haber limitaciones para que Colombia logre esa aspiración espacial.

Declaración de conflicto de interés: El autor no manifiesta conflictos de interés institucionales ni personales.

\section{Referencias}

Air Space Force Command. (s.f.). Welcome to Air Force Space Command [Bienvenido al comando espacial de la Fuerza Aérea]. Air Space Force Command. https://www.afspc. af.mil/Home/AFSPC-Mission/

Álvarez, C., Corzo, M., Jaimes, G. \& Paredes, R. (2019). La nueva economía del siglo xxI: el sector privado en el espacio. En C. Corredor \&. C. Álvarez (Eds.), El espacio exterior: Una oportunidad infinita para Colombia. Escuela Superior de Guerra “General Rafael Reyes Prieto” y Fuerza Aérea Colombiana.

Burger, E. \&. Bordacchini, G. (2017). Yearbook on space policy 2017. Security in outer space: rising for civilian space programmes. Springer.

Chung, C. K. (2001). Aerospace power in the twenty-first century: A basic primer. United States Air Force Academy; Air University Press.

Congreso de Colombia. (2018). Plan Nacional de Desarrollo 2018-2022 Pacto por Colombia, pacto por la equidad. Gobierno de Colombia.

CONPES. (2010). 3.683. Lineamientos para la formulación del programa nacional de observación de la tierra que incluya el diseño de un programa satelital colombiano. República de Colombia - Departamento Nacional de Planeación. https://colaboracion.dnp.gov.co/CDT/Conpes/ Econ\%C3\%B3micos/3683.pdf

CONPES. (2016). 3.866. Política nacional de desarrollo productivo. República de Colombia - Departamento Nacional de Planeación. https://colaboracion.dnp.gov.co/CDT/ Conpes/Econ\%C3\%B3micos/3866.pdf

CONPES. (2020). 3.983. Política de desarrollo espacial: condiciones habilitantes para el impulso de la competitividad nacional. República de Colombia - Departamento Nacional de Planeación. https://colaboracion.dnp.gov.co/CDT/ Conpes/Econ\%C3\%B3micos/3983.pdf

Fuerza Aérea Colombiana. (2020). Estrategia para el desarroIlo aéreo y espacial de la Fuerza Aérea Colombiana 2042.

Fuerza Aérea Colombiana. (2019, 28 de noviembre). FACSAT-1: Un año en el espacio. https://www.fac.mil.co/facsat-1 -un-a\%C3\%B1o-en-el-espacio

Fuerza Aérea Colombiana. (s.f.). Misión y visión Fuerza Aérea Colombiana. https://www.fac.mil.co/transparencia-yacceso-informacion-publica/3-estructura-organica-y-ta lento-humano/mision-vision\#: :text=Volar\%2C\%20en 
trenar\%20y\%20combatir\%20para,a\%20los\%20fines\% 20del\%20Estado

Joya, R. A. (s.f.). Libertad 1: Primer satélite colombiano en el espacio. Universidad Sergio Arboleda. https://www.user gioarboleda.edu.co/satelite-libertad-1/

Naciones Unidas. (1958, 13 de diciembre). Resolución 1348 (XIII). Cuestión del uso del espacio ultraterrestre con fines pacíficos. United Nations Office for Outer Space Affairs. https://www.unoosa.org/pdf/gares/ARES_13_1348S.pdf

Naciones Unidas. (2002). Tratados y principios de las Naciones Unidas sobre el espacio ultraterrestre. Oficina de Naciones Unidas para Asuntos del Espacio Exterior y Organización de las Naciones Unidas. https://www.unoosa.org/ pdf/publications/STSPACE11S.pdf

Naciones Unidas. (2015a, 10 de junio). La ONU debate sobre la inclusión del espacio exterior en la agenda de desarrollo sostenible. Centro de noticias de la ONU; Objetivos de Desarrollo Sostenible. https://www.un.org/ sustainabledevelopment/es/2015/06/la-onu-debatesobre-la-inclusion-del-espacio-exterior-en-la-agendade-desarrollo-sostenible/

Naciones Unidas. (2015b, 19 de junio). Informe de la comisión sobre la utilización del espacio ultraterrestre con fines pacíficos. $58 .^{\circ}$ periodo de sesiones. https://www.unoosa.org/res/oosadoc/data/documents/2015/a/a7020_0_ html/A_70_20S.pdf

Naciones Unidas. (2015c, 25 de septiembre). Transformar nuestro mundo: La agenda 2030 para el desarrollo sostenible. $70 .^{\circ}$ periodo de sesiones. Asamblea General. https:// unctad.org/system/files/official-document/ares70d1_ es.pdf

Naciones Unidas. (2019, 21 de junio). Informe de la Comisión sobre la Utilización del Espacio Ultraterrestre con Fines Pacíficos. $62 .^{\circ}$ periodo de sesiones. https://www.uno osa.org/res/oosadoc/data/documents/2019/a/a7420_ 0_html/V1906080.pdf

Organización para la Cooperación y el Desarrollo Económicos (OECD) (2014). The Space Economy at a Glance 2014: OECD Publishing.

Parkinson, J. (2019, octubre 8 y 10). Is fluidity the key to effective multi-domain operations? [Conference Read Ahead]. Joint air power competence centre 2019 - Shaping NATO for multi-domain operations of the future, 39-46.
Quiroga, R. G. (2019). Análisis de la política espacial colombiana: una perspectiva de defensa y seguridad. En C. Álvarez. El espacio exterior: una oportunidad infinita para Colombia, (volumen 2). (Ed.), Escuela Superior de Guerra "General Rafael Reyes Prieto" y Fuerza Aérea Colombiana.

Rodríguez, H. (2020, 16 de septiembre). Las mejores fotos históricas de la Tierra desde el espacio. National Geographic-Astronomía. https://www.nationalgeographic. com.es/ciencia/grandes-reportajes/las-mejores-fotoshistoricas-de-la-tierra-desde-el-espacio_10297

Sachs, J. (2015). La era del desarrollo sostenible. Nuestro futuro está en juego: incorporemos el desarrollo sostenible a la agenda política mundial (R. V. Vernis, Trad.). Deusto.

Tesauro de la Unesco. (2020, 3 de julio). Dominio. http://voca bularies.unesco.org/browser/thesaurus/es/search? clang $=e s \& q=$ dominio\&vocabs $=$

United Nations Office for Outer Space Affairs. (s.f.-a). Rolls and responsabilities [Funciones y responsabilidades]. https://www.unoosa.org/oosa/en/aboutus/roles-res ponsibilities.html

United Nations Office for Outer Space Affairs. (s.f.-b). Space supporting the sustainable development goals [Espacio que apoya los objetivos de desarrollo sostenible]. https://www.unoosa.org/oosa/en/ourwork/space4s dgs/index.html

United States Air Force. (2014). America's Air Force: a call to the future [Fuerza Aérea de América: un llamado al futuro]. United States Air Force. airman.dodlive.mil/fi les/2014/07/AF_30_Year_Strategy_2.pdf

UNOOSA. (2020, 7 de diciembre). Mensajes de los exploradores del espacio a las generaciones futuras. UNOOSA/documents. https://www.unoosa.org/documents/pdf/ outreach/astromessages/mseS.pdf

Urbina Carrero, J., C. (2017). El espacio, Futuro de la Fuerza Aérea Colombiana. Ciencia y Poder Aéreo, 12, 202-208. http:// dx.doi.org/10.18667/cienciaypoderaereo.572

Vicepresidencia Colombia. (2020, 15 de octubre). Palabras de la vicepresidente en la Semana Mundial del Espacio 2020 'WSW' [Video]. YouTube. https://www.youtube. com/watch?v=pLtGYbbRB3Y\&t=593s

Zacharias, G. (2019). Horizons autonomous: The way forward. Air University Press; Curtis E. LeMay Center for Doctrine Development and Education. 